\title{
Promoting an All-Inclusive Epidemiological Studies of Parkinson Disease (PD) in Ethnic Minorities of African Descent
}

\author{
Tomilowo Abijo* \\ Department of Anatomy, Howard University, USA
}

*Corresponding author: Tomilowo Abijo, Department of Anatomy, College of Medicine, Howard University, Washington DC, USA

\begin{abstract}
ARTICLE INFO
Received: 慧 January 28, 2019

Published: March 06, 2019

Citation: Tomilowo Abijo. Promoting an All-Inclusive Epidemiological Studies of Parkinson Disease (PD) in Ethnic Minorities of African Descent. Biomed J Sci \& Tech Res 15(3)-2019. BJSTR. MS.ID.002715.
\end{abstract}

Abbreviations: PD: Parkinson Disease; SN: Substantia Nigra; DA: Production of Dopamine; SS: Sub-Sahara

\begin{abstract}
According to the National Parkinson's Foundation, approximately sixty thousand Americans are diagnosed with Parkinson's Disease each year, while more than 10 million people worldwide are living with this disease. Apart from revealing important information on the pattern of spread, establishing the incidence and prevalence of Parkinson Disease along ethnic lines will provide more insight as to the genetic basis of the etiology as well as the relative risk of having Parkinson Disease. So far, available reports have shown that most epidemiological studies tend to focus more on Caucasians, especially in North Americans and Europe. This may understandably be attributed to lack of fund and resources to propagate similar studies in regions populated by other ethnic groups, especially in Africa. In this short review, we highlight the importance of extending epidemiological studies to areas that have hitherto been neglected. We also present recommendations that can potentially improve the management of Parkinson Disease in minorities, especially African-Americans.
\end{abstract}

\section{Introduction}

Parkinson Disease (PD) is a neurodegenerative disease characterized by the loss of theSubstantia Nigra(SN)accommodating dopaminergic neurons responsible for the production of dopamine (DA), an excitatory neurotransmitter, in the mid brain [1,2]. Because DA is involved in the relay of messages to the part of the brain that controls movement and coordination (Motor Cortex) via the Basal Ganglia and the Thalamus, its loss results in the complete or partial loss of movement [3]. PD symptoms can be categorized into four groups based on the individual and severity. These include tremor of the hands, arms, legs, jaw and face; bradykinesia or slowness of movement; rigidity or stiffness of the limbs and trunk; and postural instability or impaired balance and coordination [4]. It is termed a progressive neurological disorder because it worsens with time [4]. The cause of PD is not fully understood. So far, no cure has been found for PD, but certain treatments are recommended for patients based on the severity of the symptoms. Because PD's pathology is linked to loss of dopaminergic neurons that produces DA, most drugs are designed and produced as synthetic or semisynthetic DA to replenish insufficient endogenous DA in order to reverse the symptoms. The most prominent drug in this category is the levodopa, a semi-synthetic form of DA [5]. The final conversion of levodopa to DA is facilitated in the brain [6]. Although this drug and other variants have been shown to mitigate the symptoms of PD, they have not been able to reverse them completely. Apart from medications, surgical therapy, lifestyle modifications, physical therapy and change of diets are other alternative therapies that may be recommended on an individual basis [4].

\section{Etiology and Pathophysiology}

Most PD development can be traced to the loss of the SN in the mid brain (National Parkinson Foundation). The brain SN accommodates dopaminergic neurons that produce DA, an excitatory neurotransmitter that plays a critical role in movement and coordination [1,2]. Thus, the decreased production of DA results in movement disorder, characterized by tremors in people with PD [4]. Apart from the damaged or loss of SN, genetic mutations of some certain genes have been implicated in PD development. Some of these genes include PARK2 (parkin), PARK7, PINK1 (PARK6), 
LRRK2 (PARK8) and SNCA (PARK1), PARK 7(DJ-1) and PARK2 (ATP13A2) [7]. In most cases, mutations of these genes have been linked to the impairment of the SN [8], although the mechanisms are yet to be clearly defined in many cases. It is also possible that PD may be caused by mutations in some other genes that are yet to be discovered. Scientists and researchers have also discovered a correlation between PD and protein aggregates of alpha-synuclein, the major component of Lewy bodies, and a signature and marker for dementia and other neurodegenerative, neurological and cardiovascular diseases $[9,10]$. Drugs that can dissolve this protein aggregates have been developed and in preclinical testing stages [11]. While the pathology and pathophysiology of most PD development can be accounted for, about 15\% are idiopathic [12].

\section{Prevalence of PD in the United States}

Because of the lack of adequate statistics, especially in the sub-Sahara (SS), it is very difficult to state categorically how many people are living with this disease worldwide. However, according to the Parkinson's Disease Foundation, about one million people are living with this disease in the United States (US). Also, about sixty thousand new cases are detected each year minus those that go undetected. Studies have also shown that PD is about one and a half time as common in men as in women and people with a family history are $10 \%$ more likely to have it than people with no family history $[4,13]$. Although the basis for the prevalence of PD in men compared to women has not been fully understood, studies have shown that it may not be unconnected with exposure of men to toxic chemicals or head injuries, both of which have been characterized as possible PD risk factors [13]. It is also believed that estrogen may provide additional protection for females' nervous system [13]. The elucidation of the underlying mechanism of the gender-based discrimination against PD development will definitely shed more light on the pathophysiology of PD. Apart from gender, other factors that may influence PD prevalence include age, genetics, social life and family history

\section{Prevalence of PD in Ethnic Minorities in the United States}

Most data available on the prevalence and incidence of PD are based on studies done in the US, Europe and some parts of Asia. Data are lacking on the prevalence and incidence of this disease in the Sub-Saharan (SS). Although available data of studies done in the US, with the second largest population of black people after Nigeria and Brazil, showed that black people may have a low incidence of PD compared to Caucasians [14], this does not necessarily translate to the overall low prevalence of PD among black people. Many of these studies are really vulnerable to statistical biases and their outcomes have been termed unacceptable. It is necessary and important to conduct more comprehensives studies to really ascertain the prevalence of PD in minorities, especially of African descent. In Africa, the historical ancestral homeland of black minorities in the US, reliable and substantial data are lacking on the prevalence and incidence of PD. Except for South Africa, Nigeria, Ghana and Zambia, research studies both at the molecular and epidemiological levels on PD are essentially nonexistent. The unreliable epidemiological data on PD in American minorities of African descent and the lack of substantial data in Africa makes it difficult to make any substantial conclusion on the prevalence and incidence of PD between Caucasian and Blacks vis-à-vis sex, age, demography etc.

\section{Why Epidemiological Studies of PD of Minorities is Essential}

The importance of a comprehensive report on the incidence and prevalence of PD in African American, and Black people in general, cannot be overemphasized. It will definitely provide a platform to organize a comprehensive treatment scheme to manage the disease among this ethnic minority. Also, reliable epidemiological data on this disease on blacks could reveal new novel information about the genetic and environmental basis of the development and evolution of this disease. This is predicated on the fact that epidemiological studies of some diseases have revealed novel pattern of inheritance and polymorphisms, providing more information on genetic variations and diversities. Africa has been termed a dark continent when it comes to the knowledge of most neurological diseases, including PD [15]. It is generally believed PD prevalence and incidence in black people are actually more than reported in few countries where they are reported. Many factors are responsible for this development. Firstly, there is a paucity of qualified trained staff and personnel that can diagnose PD. It is projected that there will be a significant shortage of neurologists that can manage PD in the US by 2025 if the present rate of turnover is not increased [16]. Secondly, the reluctance of people to participate in clinical trials and epidemiological studies. Cultural beliefs, religious sentiments and prejudice, especially in Africa, may also discourage people with PD from coming out to declare their status.

\section{Correcting PD Management Disparities in African Americans}

\section{Comprehensive Epidemiological Studies}

The first step in correcting the disparities of the management of PD in African American minorities is to conduct comprehensive epidemiological studies on this group of people. While comprehensive epidemiological studies of this disease are also recommended in the sub-Saharan to provide a comprehensive conclusion on the prevalence and incidence of this disease in black people as a whole, the financial and demographic burdens may be quite challenging. Therefore, for a start, the studies can be limited to Africans in the United States and the research extended to the subSaharan in the future. The epidemiological studies must emphasize the frequencies and pattern of PD distribution. The frequencies studies must emphasize the prevalence and incidence with respect to time, space, age, gender and social status. 


\section{Clinical and Genetic studies (Genome Wide Association Studies)}

African Americans should be encouraged to participate in large scale screenings to identify potential gene mutations which could results in PD development. These genes include PARK2 (parkin), PARK7, PINK1 (PARK6), LRRK2 (PARK8) and SNCA (PARK1), PARK 7(DJ-1) and PARK2 (ATP13A2). More genetic studies are recommended for African Americans in order to identify novel genes mutations that can also cause PD. Genetics studies is a powerful tool that can reveal a lot of information about the vulnerability and susceptibility to a disease vis-à-vis sex, age, race and demography. For instance, genetic studies have shown that female ethnic minorities of African descent are vulnerable to having and dying from certain types of breast cancer compared to their Caucasian counterpart [17]. In addition, novel race-specific polymorphisms, including single nuclear polymorphisms and copy number polymorphisms can be identified and patients can be advised in advance of their chances of having PD.

\section{Periodic Screening}

Periodic screenings should be encouraged among minorities to access and examine their health conditions and their propensity to having PD. This screening may reveal past family histories of PD. People with family histories of PD have about ten chances of having PD compared to people with no family histories.

\section{Affordable Health Care Provision}

Although the government is doing a lot to make health care affordable to patients, there is still need for improvement especially with regard to minorities. This is predicated on the facts that most minorities, the majority of whom live below the middle class, cannot afford the minimum health insurance plans. Also, as PD requires a medical specialist attention, copayment should be waived by the Insurance companies. Many patients may be forced to hide their family histories of PD to avoid being classified as preexisting by the insurance companies since most preexisting classified patients are rejected by insurance companies or in the worst scenario case accepted with a high premium insurance rate which may be unaffordable to the patients. The implications of hiding a family history of a disease, including PD, is that it prevents early intervention measures. This should not only be discouraged but should be discontinued.

\section{Individual Focused Interventions}

There is a general misconception that health care disparities among ethnic groups can be eradicated by simply improving the quality of health care of the general population. Past studies have shown that this may not be true after all. A 15-year progress analysis of carried out to investigate black-white health disparities in the United States vis-à-vis improved general quality of health in Chicago area from 1990 to 2005 showed that rather than closing the health disparities gap, it was further widened, although the qualities of health care for the two groups were significantly improved [18]. A recent study showed that individual-focused interventions may be very effective in managing chronic diseases. This is based on the premise that it provides a combination of prevention interventions that consider individual lifestyle, genetics and environment rather than general characteristics [19]. Individually focused intervention should also be employed in the management of PD.

\section{Sensitization of Local Communities}

Also, efforts should be directed at increasing the number of health care providers, especially medical specialists in areas that are densely populated by minorities. Studies have shown that minorities with terminal diseases, including PD, are less likely to be accepted by specialists due to prejudice about their ability to pay. To prevent this situation, government and private agencies should encouraged private care providers to assist and educate minorities, especially those with low income on the need to enroll in the Medicare program of the government. They should be made to understand that been healthy at all time is a personal responsibility and no effort should be spared in getting access to adequate treatment, even when the patients cannot afford it.

\section{Clinical Trials}

Minorities should be encouraged to participate in clinical trials of potential new drugs for PD treatment. Because PD affects a relatively small number of people, pre-clinical trials to identify new drugs for its treatment has proven relatively difficult. Although studies have shown that a lot of minorities are skeptical about clinical studies and their outcomes, these sentiments and prejudice can be eradicated through proper education and sensitization about the importance of clinical trials in the management of diseases, including PD [20]. The idea of monetary compensation to encourage participation should also be encouraged.

\section{References}

1. Lang AE, Lozano AM (1998) Parkinson's disease. Second of two parts. N Engl J Med 339(16): 1130-1143.

2. Kim SJ, Sung JY, Um JW, Hattori N, Mizuno Y, et al. (2003) Parkin Cleaves Intracellular -Synuclein Inclusions via the Activation of Calpain. Journal of Biological Chemistry 278(43): 41890-41899.

3. Dirkx MF, den Ouden EM, Aarts E, Timmer HM, Bloem BR, et al. (2017) Dopamine controls Parkinson's tremor by inhibiting the cerebellar thalamus. Brain 140(3): 721-734.

4. Parkinson Disease Foundation.

5. Brooks DJ (2008) Optimizing levodopa therapy for Parkinson's disease with levodopa/carbidopa/entacapone: implications from a clinical and patient perspective. Neuropsychiatr Dis Treat 4(1): 39-47.

6. Koller WC, Rueda WC (1998) Mechanism of action of dopaminergic agents in Parkinson's disease. Neurology 50(6 Suppl 6): S11-14, discussion S44-S48.

7. Klein C, Westenberger A (2012) Genetics of Parkinson's Disease. Cold Spring Harb Perspect Med 2(1): a008888.

8. Mark R Cookson, John Hardy, Patrick A Lewis (2008) Genetic Neuropathology of Parkinson's Disease Int J Clin Exp Pathol 1(3): 217231. 
9. Spillantini MG, Crowther RA, Jakes R, Hasegawa M, Goedert M (1998) alpha-Synuclein in filamentous inclusions of Lewy bodies from Parkinson's disease and dementia with lewy bodies. Proc Natl Acad Sci USA 95(11): 6469-6473.

10. Braak H, Del Tredici K, Rüb U, de Vos RA, Jansen Steur EN, et al. (2003) Staging of brain pathology related to sporadic Parkinson's disease. Neurobiol Aging 24(2): 197-211.

11. (2014) Parkinson's Vaccine: EU-Team Launches Clinical Trial Vaccine candidate based on proprietary technology by AFFiRiS AG. Vienna, Europe.

12. National Parkinson Foundation.

13. Wooten GF, Currie LJ, Bovbjerg VE, Lee JK, Patrie J (2004) Are men at greater risk for Parkinson's disease than women? J Neurol Neurosurg Psychiatry 75(4): 637-639.

14. Dahodwala N, Siderowf A, Xie M, Elizabeth Noll, Matthew Stern, et al. (2009) Racial Differences in the Diagnosis of Parkinson's Disease. Mov Disord 24(8): 1200-1205.

\section{ISSN: 2574-1241}

DOI: 10.26717/BJSTR.2019.15.002715

Tomilowo Abijo. Biomed J Sci \& Tech Res

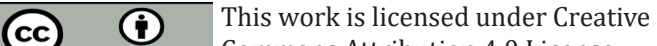

Submission Link: https://biomedres.us/submit-manuscript.php
15. Pearce V, Wilson I (2007) Parkinson's disease in Africa. Age Ageing 36(2): 116-117.

16. Dall TM, Storm MV, Chakrabarti R, Drogan O, Keran, BA, et al. (2013) Supply and demand analysis of the current and future US neurology workforce. Neurology 81(5): 470-478.

17. O'Keefe EB, Meltzer JP, Bethea TN (2015) Health Disparities and Cancer: Racial Disparities in Cancer Mortality in the United States, 2000-2010. Front Public Health 3: 51.

18. Orsi JM, Margellos-Anast H, Whitman S (2010) Black-White Health Disparities in the United States and Chicago: A 15-Year Progress. Analysis American Journal of Public Health 100(2): 349-356.

19. Saeed S, Golfam M, Beall RF, Ashbury FD, Palmer LJ, et al. (2014) Effectiveness of individual-focused interventions to prevent chronic disease. European Journal of Clinical Investigation 44(9): 883-891.

20. Branson RD, Davis K Jr, Butler KL (2007) African Americans' participation in clinical research: importance, barriers, and solutions. Am J Surg 193(1): 32-39.

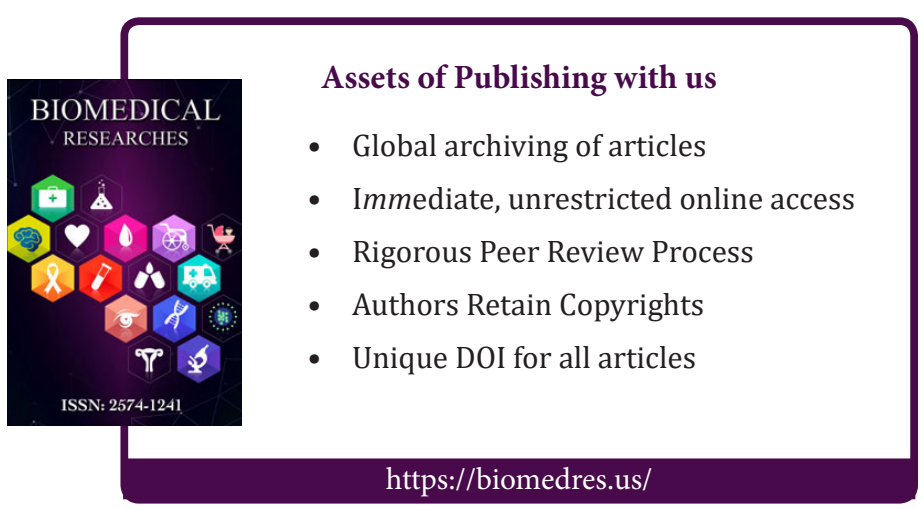

